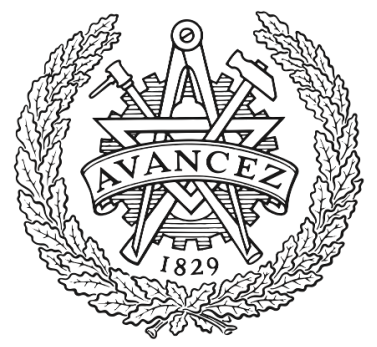

CHALMERS

UNIVERSITY OF TECHNOLOGY

\title{
Location-Aided Pilot Contamination Avoidance for Massive MIMO Systems
}

Downloaded from: https://research.chalmers.se, 2023-04-26 12:20 UTC

Citation for the original published paper (version of record):

Muppirisetty, S., Charalambous, T., Karout, J. et al (2018). Location-Aided Pilot Contamination Avoidance for Massive MIMO Systems. IEEE Transactions on Wireless Communications, 17(4): 2662-2674. http://dx.doi.org/10.1109/TWC.2018.2800038

N.B. When citing this work, cite the original published paper.

C2018 IEEE. Personal use of this material is permitted.

However, permission to reprint/republish this material for advertising or promotional purposes 


\title{
Location-Aided Pilot Decontamination for Massive MIMO Systems
}

\author{
L. Srikar Muppirisetty, Themistoklis Charalambous, Member, IEEE, Johnny Karout, Senior Member, IEEE, \\ Gábor Fodor, Senior Member, IEEE, and Henk Wymeersch, Member, IEEE
}

\begin{abstract}
One of the key limitation of massive MIMO systems is pilot contamination, which is defined as the interference during uplink channel estimation due to re-use of the same pilots in surrounding cells. In this paper, we propose a location-based approach to the pilot contamination problem for uplink MIMO systems. Our approach makes use of the approximate locations of mobile devices to provide good estimates of the channel statistics between the mobile devices and their corresponding base stations (BSs). We aim at minimizing the pilot contamination even when the number of $\mathrm{BS}$ antennas is not very large, and when multiple users from different cells, or even the same cell, are assigned the same pilot sequence. First, we characterize a desired angular region of the target user at the target $\mathrm{BS}$ in which interference is very low or zero, based on the number of BS antennas and the location of the target user. Second, based on this observation, we propose various pilot coordination methods for multi-user multi-cell scenarios to eliminate pilot contamination.
\end{abstract}

Index Terms-Interference alignment, MIMO systems, pilot contamination, location-aware communication.

\section{INTRODUCTION}

The use of very large antenna arrays at the base station (BS) is considered as a promising technology for 5G communications in order to cope with the increasing demand of wireless services [1]. Such massive multi-input multi-output (MIMO) systems provide numerous advantages [2]-[6]: (i) they increase spectral efficiency by supporting a higher number of users per cell, (ii) they improve energy efficiency by radiating focused beams towards users, and (iii) they offer channel hardening effect, where small-scale fading is averaged out. Furthermore, under the assumption of perfect channel estimation, massive MIMO provides asymptotic orthogonality between vector channels of the target and interfering users. However, the performance of these systems is limited by the pilot contamination effect, i.e., interference during uplink channel estimation due to re-use of the same pilot sequences.

Pilot sequences are a scarce resource due to the fact that the length of pilot sequences (number of symbols) is limited by the coherence time and bandwidth of the wireless channel. As a result, the number of separable users is limited by the number of the available orthogonal pilot sequences [4], [5]. Therefore, in multi-cell massive MIMO systems, the pilot sequences must

L. Srikar Muppirisetty, Themistoklis Charalambous and Henk Wymeersch are with the Department of Signals and Systems, Chalmers University of Technology, 41296 Gothenburg, Sweden, e-mail: \{srikar.muppirisetty, themistoklis.charalambous, henkw\} @ chalmers.se.

Johnny Karout and Gábor Fodor are with Ericsson Research, Kista, 16480 Stockholm, Sweden, email:\{johnny.karout, gabor.fodor\}@ericsson.com.

This research was supported, in part, by the European Research Council, under Grant No. 258418 (COOPNET). be reused, which leads to interference between identical pilot sequences from users in either neighboring cells or even the same cell; this effect is known as pilot contamination [7]. Pilot contamination is known to degrade the quality of channel state information at the $\mathrm{BS}$, which in turn degrades the performance in terms of the achieved spectral efficiency, beamforming gains, and cell-edge user throughput.

Mitigation strategies for pilot contamination have been well studied in the literature. Existing approaches include, among others, blind channel estimation using eigenvaluedecomposition [8], greater-than-one pilot reuse schemes [9], modifying the frame structure such that the pilots are transmitted in each cell in non-overlapping time slots [10], [11], transmitting pilots in consecutive phases in which each BS keeps silent in one phase and repeatedly transmits in other phases [12], mixture of downlink and uplink training [13], and exploiting second-order statistics of desired and interfering user channels [14]. In particular, [14] exploited the covariance matrices of the desired and interfering channels and showed that by carefully allocating the pilots to the users, the pilot contamination effect is eliminated in the large antenna array regime. The design is based on the knowledge of the covariance matrices of the desired channel, as well as the interfering channels. These covariance matrices scale roughly quadratically with the number of BS antennas per user and thus may be impractical to estimate. In our previous work [15], a location-aided approach is proposed, in which the scalability problem in estimating covariance matrices [14] is eliminated. In particular, the mean and the standard deviation of the angleof-arrival (AoA) are related to a user location, rather than to a user's channel.

The works in [14], [15] showed that pilot contamination is eliminated when the number of BS antennas goes to infinity. However, it is possible that the number of antennas can be limited such that the pilot contamination effect does not vanish. Furthermore, the pilot assignment proposed in [14], [15] considers the mitigation of pilot contamination only one user at a time in the target cell. Hence, after a set of users has been assigned a certain pilot, the process is repeated for another user in the target cell, and so forth. This is a greedy approach, which favors the first user, and later users suffer from lack of options, as there will be fewer users from the other cells to choose from. This performance degradation due to greedy pilot allocation has been reported in the literature (see for example, [9]). Furthermore, the assignment aims at reducing the interference seen by the target cell users only, with no regard to the interference that users in the neighboring 
cells experience.

In this work, we address the aforementioned issues by considering a joint design across multiple cells for all users in the system when the number of BS antennas is not very large. More specifically, the contributions of our paper are as follows:

- We first propose an approach with which, in the presence of location information of the users, we can quantify the effect of pilot contamination for BSs with a finite number of antennas. This result helps us predict how each user interferes with the rest of the users having identical pilot sequences when we have BSs with MIMO antennas, and the number of antennas is not necessarily approaching infinity. This quantification reveals that for a considerable number of antennas, there is a range of angles for which the pilot contamination is very small.

- Based on the above observation, we formulate pilot decontamination as an integer quadratic programming problem that we are able to solve for all the BSs as a joint optimization problem. In particular, we propose multi-cell multi-user joint optimization problems such that it takes into consideration during the pilot assignment the mutual interference seen by the target users at their respective BSs.

The rest of the paper is structured as follows. In Section II, we introduce the system model comprising the network model, the channel model, and the received pilot signal. In Section III, we provide a brief overview of the uplink channel estimation via a minimum mean square error (MMSE) estimator. In Section IV, we address the problem of pilot decontamination for BSs with (not necessarily massive) MIMO antennas. In Section V, we present optimal user assignment strategies for pilot decontamination under various configurations, building on the theory developed in Section IV. In Section VI, we demonstrate the performance of the proposed methods and we compare them with other user selection methods proposed in the literature. Finally, in Section VII we draw conclusions and discuss possible future directions.

\section{Notation}

Throughout the paper, vectors are written in bold lower case letters and matrices in bold upper case letters. For matrix $\mathbf{X}$, matrices $\mathbf{X}^{\mathrm{T}}$ and $\mathbf{X}^{\mathrm{H}}$ denote its transpose and hermitian, respectively. The $i$-th entry of vector $\mathbf{x}$ is denoted as $[\mathbf{x}]_{i}$. $\operatorname{vec}[\mathbf{X}]$ denotes stacking all the elements of $\mathbf{X}$ in a vector. $U[\mathcal{S}]$ denotes a uniform distribution over the intervals defined by the set $\mathcal{S}$. We define a rotation operator $\mathcal{R}_{\theta_{2}}\left(\theta_{1}\right)=$ $\bmod \left(\theta_{1}-\theta_{2}, \pi\right)$, in which $\theta_{1}$ is the input and $\theta_{2}$ is the parameter of the operator. The operator $\mathcal{R}_{\theta_{2}}\left(\theta_{1}\right)$ rotates a vector with orientation $\theta_{1}$ in clockwise direction by $\theta_{2}$, followed by angle wrapping such that $\mathcal{R}_{\theta_{2}}\left(\theta_{1}\right) \in[0, \pi]$. A sequence of elements $\left\{a_{1}, a_{2}, \ldots\right\}$ is written in short as $\left\{a_{j}\right\}_{j}$. The positive operator is denoted as $(x)^{+}=\max (0, x)$. The Kronecker product of two matrices $\mathbf{X}_{1}$ and $\mathbf{X}_{2}$ is denoted as $\mathbf{X}_{1} \otimes \mathbf{X}_{2}$. $\mathbf{I}_{M}$ denotes the identity matrix of size $M \times M$ and $\|\cdot\|_{2}$ denotes the Euclidean norm. The cardinality of a set $\mathcal{A}$ is denoted by $|\mathcal{A}|$. The sets of real and complex numbers are denoted by $\mathbb{R}$ and $\mathbb{C}$, respectively; the $n$-dimensional Euclidean and complex spaces are denoted by $\mathbb{R}^{n}$ and $\mathbb{C}^{n}$, respectively.

\section{SySTEM MODEL}

\section{A. Network model}

We consider a two-dimensional scenario with cells, and each cell is served by one BS equipped with $M$ antennas. We denote $\mathcal{C}$ as the set of all cells and where $\mathcal{K}_{j}$ the set of users in the $j$-th cell, $j \in \mathcal{C}$. The set of neighboring cells to the $j$-th cell is denoted by $\mathcal{C}_{j}^{\text {sur }}$. Users, equipped with a single antenna, are located uniformly within the cells. The location of user $i \in \mathcal{K}_{j}$ is denoted by $\mathbf{x}_{i j} \in \mathbb{R}^{2}$, while the location of the BS in the $k$-th cell is written as $\mathbf{x}_{k} \in \mathbb{R}^{2}$. We define $\mathcal{P}$ as the set of available orthogonal pilots for allocation to users.

\section{B. Channel Model}

The uplink channel of user $i$ from cell $j$ to BS $k$ is denoted by $\mathbf{h}_{i j k} \in \mathbb{C}^{M}$. We note that the channel depends only on user $i$ and $\mathrm{BS} k$, but the use of the additional index $j$ allows us to distinguish in which cell the users belong to. The channel is modeled as the superposition of $B$ arriving paths [14], [16]

$$
\mathbf{h}_{i j k}=\frac{1}{\sqrt{B}} \sum_{b=1}^{B} \mathbf{a}\left(\theta_{i j k}^{(b)}\right) \alpha_{i j k}^{(b)}
$$

where $\mathbf{a}\left(\theta_{i j k}\right) \in \mathbb{C}^{M}$ is the antenna steering vector corresponding to AoA $\theta_{i j k} \in[0,2 \pi), b$ is the path index, and $\alpha_{i j k}^{(b)}$ is the channel coefficient of the $b$-th path. The AoA's are assumed to be i.i.d., with probability density $p\left(\theta_{i j k}\right)$. We model $\alpha_{i j k}^{(b)} \stackrel{\text { i.i.d. }}{\sim} \mathcal{C N}\left(0, \beta_{i j k}\right)$, in which $\beta_{i j k}$ depends on pathloss and shadowing between user $i$ in cell $j$ and BS $k$. In particular, let $\alpha$ be a constant that depends on cell-edge signalto-noise ratio (SNR) and cell radius $R$. It is defined as

$$
\alpha[\mathrm{dB}]=\gamma_{\mathrm{SNR}}+10 \eta \log _{10}(R)+10 \log _{10}\left(\sigma^{2}\right),
$$

where $\gamma_{\mathrm{SNR}}$ is the cell-edge SNR in $\mathrm{dB}, \eta$ is the path-loss exponent, and $\sigma^{2}$ is the receiver noise power variance. Then, in linear scale $\beta_{i j k}=\alpha\left\|\mathbf{x}_{i j}^{\mathrm{u}}-\mathbf{x}_{k}^{\mathrm{b}}\right\|_{2}^{-\eta}$. By restricting ourselves to uniform linear arrays, the $m$-th entry in the steering vector is given by [14]

$$
\left[\mathbf{a}\left(\theta_{i j k}\right)\right]_{m}=\exp \left(-j 2 \pi m D \cos \left(\theta_{i j k}\right) / \lambda\right),
$$

in which $D$ is the antenna spacing at the BS and $\lambda$ is the signal wavelength. It should be noted that we can limit $\theta_{i j k} \in[0, \pi]$ because any angle $\theta_{i j k} \in[-\pi, 0]$ can be replaced by $-\theta_{i j k}$, thus giving the same steering vector.

\section{Received Pilot Signal}

We consider a scenario, in which the target user $i$ in cell $k$ sending uplink transmission to BS $k$. We assume users from various cells have been assigned the same pilot sequence $\mathbf{s}$ of length $\tau$. For notational convenience, we will further assume that all the users indexed with $i$ are also assigned the same pilot sequence s. Later, in Section V, we will present various ways to assign users across cells to a given pilot sequence. 


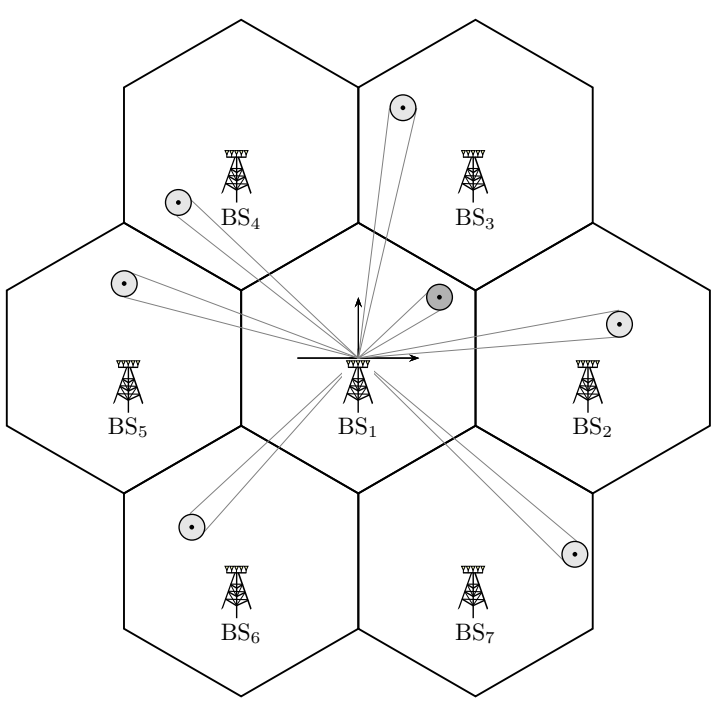

(a)

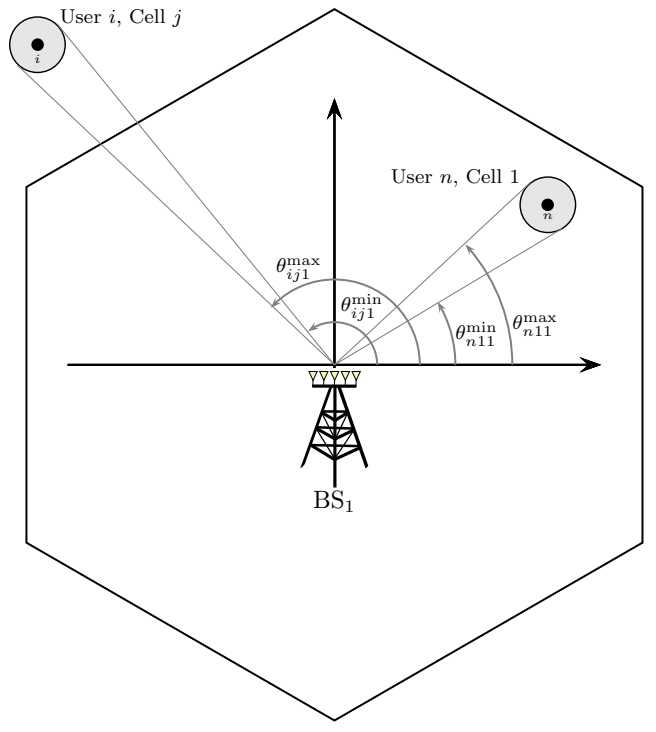

(b)

Fig. 1. (a) A two-dimensional 7-cell hexagonal layout with a BS placed at the center of each cell. The users in each cell are marked with a black dot and the scatterers lie within a circle around the user; (b) The support of AoA of desired and interfering user at the target BS and shows a specific example with desired user $n$, interfering user $i$ from cell $j$. The radius of the ring (shaded region around the user location) of scatterers is $r_{\mathrm{s}}$. The supports of the desired and interfering users $\mathrm{AoA}$ at the target $\mathrm{BS}$ are $\left[\theta_{n 11}^{\min }, \theta_{n 11}^{\max }\right]$ and $\left[\theta_{i j 1}^{\min }, \theta_{i j 1}^{\max }\right]$, respectively.

The received $M \times \tau$ pilot signal observed at $\mathrm{BS} k$ is written as

$$
\mathbf{Y}_{k}=\mathbf{h}_{i k k} \mathbf{s}^{\mathrm{T}}+\sum_{j \in \mathcal{C}} \mathbf{h}_{i j k} \mathbf{s}^{\mathrm{T}}+\mathbf{N},
$$

where $\mathbf{N} \in \mathbb{C}^{M \times \tau}$ is spatially and temporally additive white Gaussian noise (AWGN) with element-wise variance $\sigma^{2}$. In (4), $\mathbf{h}_{i k k}$ is the desired signal channel in the cell $k$ and $\mathbf{h}_{i j k}$ are the channels of interfering users.

\section{UPLINK ChANNEL ESTIMATION}

In this section, we review the MMSE estimator for the uplink channel estimate of the desired channel in the presence of interfering signals from the various cells. The estimator depends on the second order statistics of the desired and interfering channels. We also present a method based on location information of the users to compute necessary covariance matrices for the estimator.

\section{A. MMSE Channel Estimator}

The MMSE estimate of the desired channel $\mathbf{h}_{i k k}$ by BS $k$ is given by [14, Eq. (18)]

$$
\hat{\mathbf{h}}_{i k k}=\mathbf{R}_{i k k}\left(\sigma^{2} \mathbf{I}_{M}+\tau\left(\mathbf{R}_{i k k}+\sum_{j \in \mathcal{C}} \mathbf{R}_{i j k}\right)\right)^{-1} \overline{\mathbf{S}}^{\mathrm{H}} \operatorname{vec}\left[\mathbf{Y}_{k}\right],
$$

where $\overline{\mathbf{S}}=\mathbf{s} \otimes \mathbf{I}_{M}$ and $\mathbf{R}_{i j k} \in \mathbb{C}^{M \times M}$ is the covariance matrix of $\mathbf{h}_{i j k}$. The estimator (5) critically relies on the knowledge of the covariance matrices. Due to the high-dimensionality of $\mathbf{R}_{i j k}$, this information is difficult to obtain in practice. When $\mathbf{R}_{i j k}$ is perfectly known to BS $k$, intelligent pilot assignment can be performed to minimize the effect of pilot contamination (see, for example, [14]). The channel estimate of the desired channel in the presence of no interfering pilot signals from other cells can be obtained by setting the interference terms to zero in (5), leading to the estimate

$$
\hat{\mathbf{h}}_{i k k}^{\text {no-int }}=\mathbf{R}_{i k k}\left(\sigma^{2} \mathbf{I}_{M}+\tau \mathbf{R}_{i k k}\right)^{-1} \overline{\mathbf{S}}^{\mathrm{H}} \mathbf{y}_{k},
$$

where $\mathbf{y}_{k}=\overline{\mathbf{S}} \mathbf{h}_{i k k}+\operatorname{vec}[\mathbf{N}]$ is the received $\tau \times 1$ pilot signal vector at the BS $k$ under no interference from the other cell users.

\section{B. Location-Aided Covariance Estimation}

For the channel model considered in this work (see Section II-B), the covariance estimation is given by

$$
\begin{aligned}
\mathbf{R}_{i j k} & =\mathbb{E}\left[\mathbf{h}_{i j k}\left(\mathbf{h}_{i j k}\right)^{\mathrm{H}}\right] \\
& =\frac{1}{B} \sum_{b=1}^{B} \sum_{b^{\prime}=1}^{B} \mathbb{E}\left[\mathbf{a}\left(\theta_{i j k}^{(b)}\right) \mathbf{a}^{\mathrm{H}}\left(\theta_{i j k}^{\left(b^{\prime}\right)}\right)\right] \mathbb{E}\left[\alpha_{i j k}^{(b)}\left(\alpha_{i j k}^{\left(b^{\prime}\right)}\right)^{\mathrm{H}}\right] \\
& =\frac{\beta_{i j k}}{B} \sum_{b=1}^{B} \mathbb{E}\left[\mathbf{a}\left(\theta_{i j k}^{(b)}\right) \mathbf{a}^{\mathrm{H}}\left(\theta_{i j k}^{(b)}\right)\right]
\end{aligned}
$$

If we assume the AoA's are drawn from a distribution $p\left(\theta_{i j k}\right)$, then this further simplifies to

$$
\mathbf{R}_{i j k}=\beta_{i j k} \int_{0}^{2 \pi} p\left(\theta_{i j k}\right) \mathbf{a}\left(\theta_{i j k}\right) \mathbf{a}^{\mathrm{H}}\left(\theta_{i j k}\right) \mathrm{d} \theta_{i j k} .
$$

The distribution $p\left(\theta_{i j k}\right)$ is governed by the physical propagation environment. In some scenarios, as in [17, Fig. 1], propagation can be dominated by scatterers in the vicinity of the users. As an approximation, we consider a disk of radius $r_{i j k}$ comprising many scatterers around the user $i$ in cell $j$. This radius can be different for each user depending on the environment. Throughout this work, we assume that these radii are constant and, without loss of generality, we assume they 
are all equal and we denote this radius by $r_{\mathrm{s}}$. In that case, $p\left(\theta_{i j k}\right)$ corresponds to a distribution with support $\left[\theta_{i j k}^{\min }, \theta_{i j k}^{\max }\right]$, for some fixed $\theta_{i j k}^{\min }, \theta_{i j k}^{\max } \in[0,2 \pi], \theta_{i j k}^{\min }<\theta_{i j k}^{\max }$. We can calculate $\theta_{i j k}^{\min }=\theta_{i j k}^{\mu}-\theta_{i j k}^{\delta}, \theta_{i j k}^{\max }=\theta_{i j k}^{\mu}+\theta_{i j k}^{\delta}$, where

$$
\begin{aligned}
& \theta_{i j k}^{\mu}=\arctan \left(\frac{\left[\mathbf{x}_{i j}\right]_{2}-\left[\mathbf{x}_{k}\right]_{2}}{\left[\mathbf{x}_{i j}\right]_{1}-\left[\mathbf{x}_{k}\right]_{1}}\right), \\
& \theta_{i j k}^{\delta}=\arcsin \left(\frac{r_{\mathrm{s}}}{\left\|\mathbf{x}_{i j}-\mathbf{x}_{k}\right\|_{2}}\right) .
\end{aligned}
$$

Combined with the knowledge of $\beta_{i j k}$, which can also be related to the user's position since $\beta_{i j k}=\alpha\left\|\mathbf{x}_{i j}-\mathbf{x}_{k}\right\|_{2}^{-\eta}$, it is then possible to numerically compute $\mathbf{R}_{i j k}$. Hence, given the location of the desired and interfering users, BS $k$ can infer the covariance matrices and utilize those to compute $\hat{\mathbf{h}}_{i k k}$ in (5).

Remark 1. We consider a Line-of-Sight (LOS) scenario in which, nevertheless, there might be local scatterers resulting to small angular spreads. However, there might be cases where the scatterers are strong, causing large angular spreads, or cases where the location of users is not accurately known (i.e., the location estimates have uncertainty). These aspects can be incorporated into the system by allowing a larger radius $r_{\mathrm{s}}$, which translates to a larger range of angles. More complex AoA distributions can also be used by means of multiple scattering rings.

\section{Pilot Decontamination}

In this section, we discuss pilot decontamination methods under massive and finite antenna array setting. For legibility, the extra subscripts are dropped. We consider the scenario of a given target user, say user $i$, with channel $\mathbf{h}_{i}$ and associated AoA distribution $\left[\theta_{i}^{\min }, \theta_{i}^{\max }\right]$. Our objective is to find an interfering user, say user $j$, in the surrounding cells and assign it the same pilot sequence as user $i$. These users have AoAs in the ranges $\left\{\left[\theta_{j}^{\min }, \theta_{j}^{\max }\right]\right\}_{j}$ with respect to (w.r.t.) target BS for the corresponding channels $\left\{\mathbf{h}_{j}\right\}_{j}$. It will turn out to be convenient to rotate all angles with respect to user $i$. In particular, we apply the angle transformation $\mathcal{R}_{\theta_{i}^{\mu}}\left(\theta_{i}^{\mu}\right)$, so that $\theta_{i}^{\mu}$ is the new zero-degrees axis. The corresponding modified AoA supports of the desired and interfering user after angle transformation are $I_{i}^{(i)}$ and $I_{j}^{(i)}$ respectively. Furthermore, we denote $\theta_{i}^{(i)} \in I_{i}^{(i)}$ and $\theta_{j}^{(i)} \in I_{j}^{(i)}$. The subscript denotes the user index and the superscript indicates with respect to which user the angle transformation has been applied.

\section{A. Massive MIMO}

For a massive antenna array setting, it has been shown in [14, Theorem 1] that when the intervals $\left\{I_{j}^{(i)}\right\}_{j}$ are strictly non-overlapping with $I_{i}^{(i)}$, i.e., $I_{j}^{(i)} \cap I_{i}^{(i)}=\emptyset, \forall j \neq i$, then

$$
\lim _{M \rightarrow \infty} \hat{\mathbf{h}}_{i}=\hat{\mathbf{h}}_{i}^{\text {no-int }} \text {. }
$$

Thus, when the number of antennas goes to infinity, the pilot contamination is eliminated completely and the channel estimate of the target user reaches interference-free scenario. The setup is visualized in Fig. 1, depicting the location of users, BSs, disc of scatterers, and the description of the two user's AoA support.

We now consider approaches based on location information for user assignment such that the pilot contamination is minimized with an increase in number of BS antennas. The approach for user assignment when $M$ is finite is discussed in the following section.

\section{B. Finite $M I M O$}

In the finite antenna regime, we can make the following claims, regarding the estimate $\hat{\mathbf{h}}_{i}$ in the presence of interfering users:

1) If two users are at the same distance to the target BS, but with different AoAs, then the user $j$ for which $\mathbf{a}\left(\theta_{j}\right)^{\mathrm{H}} \mathbf{R}_{i} \mathbf{a}\left(\theta_{j}\right)$ is the smallest, will contribute least to the interference. The reason is outlined in Appendix A.

2) If two users have the same AoA with respect to the target $\mathrm{BS}$, but are at different distances, then the furthest user will contribute least the interference. This is because the path-loss for the further user is higher. In addition, users $j$ further away will have smaller sets $I_{j}^{(i)}$. Hence, selecting users based on the AoA will automatically prefer users further away. Thus, we can focus exclusively on identifying users with small $\mathbf{a}(\theta)^{\mathrm{H}} \mathbf{R}_{i} \mathbf{a}(\theta)$.

Following the approach from [14] which is detailed for $M \rightarrow$ $\infty$, we carry out the analysis for finite $M$. Let us consider the desired user AoA after angular transformation is bounded by $p\left(\theta_{i}^{(i)}\right)$ and $\mathbf{R}_{i}$ is its covariance matrix, and consider a set of angles $\Phi$ which is not in the support set of $p\left(\theta_{i}^{(i)}\right)$. Consider the minimization

$$
\begin{aligned}
& \min _{\phi \in \Phi} \frac{1}{M} \mathbf{a}(\phi)^{\mathrm{H}} \mathbf{R}_{i} \mathbf{a}(\phi) \\
& =\min _{\phi \in \Phi} \frac{1}{M} \mathbb{E}\left[\left|\mathbf{a}(\phi)^{\mathrm{H}} \mathbf{a}\left(\theta_{i}^{(i)}\right)\right|^{2}\right] \\
& =\min _{\phi \in \Phi} \frac{1}{M} \int J^{2}\left(\theta_{i}^{(i)}, \phi\right) p\left(\theta_{i}^{(i)}\right) \mathrm{d} \theta_{i}^{(i)},
\end{aligned}
$$

where $J\left(\theta_{i}^{(i)}, \phi\right)=\mid \sum_{m=1}^{M} \exp \left(2 \pi j(m-1) \frac{D}{\lambda}(\cos (\phi)-\right.$ $\left.\left.\cos \left(\theta_{i}^{(i)}\right)\right)\right) \mid$.

Note that in [14], it is shown that when $M \rightarrow \infty$, (12c) approaches zero. As a result the interference from the interfering users is eliminated in large antenna regime, thereby the desired channel estimate approaches to the interferencefree channel estimate.

Proposition 2. The minimization $\min _{\phi \in \Phi} \frac{1}{M} \mathbf{a}(\phi)^{\mathrm{H}} \mathbf{R}_{i} \mathbf{a}(\phi)$ (12a) is equivalent to point-wise minimization of

$$
\min _{\phi \in \Phi} J\left(\theta_{i}^{(i)}, \phi\right) p_{i}\left(\theta_{i}^{(i)}\right)
$$

Proof: See Appendix B.

1) Uniform distribution: By assuming that the probability distribution $p\left(\theta_{i}^{(i)}\right)$ is uniform (i.e., $\theta_{i}^{(i)} \sim U\left[I_{i}^{(i)}\right]$ ), then for any $\theta_{i}^{(i)} \in I_{i}^{(i)}$, the minimization problem in (13) becomes $\min _{\phi \in \Phi} J\left(\theta_{i}^{(i)}, \phi\right)$. Using the finite sum of a geometric series, 


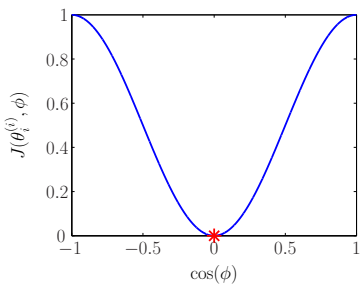

(a)

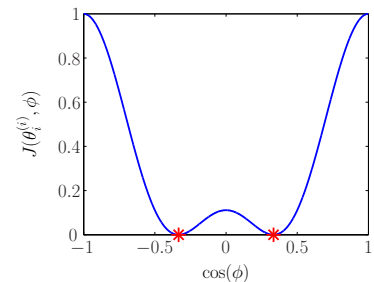

(b)

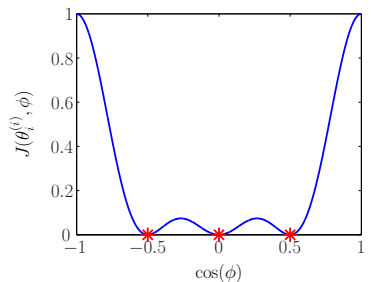

(c)

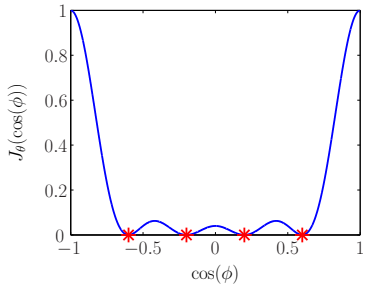

(d)

Fig. 2. The behavior $J\left(\theta_{i}^{(i)} \phi\right)$ with $\cos (\phi)$, for $\theta_{i}^{(i)}=0$ and for various antenna array lengths: (a) $M=2$, (b) $M=3$, (c) $M=4$, (d) $M=5$. The zeros $\left.\left\{\cos \left(\phi_{r}^{*}\right)\right\}_{r}\right\}$ are depicted with red asterisks $(*)$.

we obtain

$$
J\left(\theta_{i}^{(i)}, \phi\right)=\frac{\left|1-\exp \left(2 \pi j M \frac{D}{\lambda}\left(\cos (\phi)-\cos \left(\theta_{i}^{(i)}\right)\right)\right)\right|}{\left|1-\exp \left(2 \pi j \frac{D}{\lambda}\left(\cos (\phi)-\cos \left(\theta_{i}^{(i)}\right)\right)\right)\right|} .
$$

Under uniform distribution, the denominator never becomes zero, since $\phi \notin I_{i}^{(i)}$.

It can be easily deduced that the minimum of $J\left(\theta_{i}^{(i)}, \phi\right)$ is 0 and is attained when the numerator becomes zero, i.e.,

$$
\exp \left(2 \pi j M \frac{D}{\lambda}\left(\cos (\phi)-\cos \left(\theta_{i}^{(i)}\right)\right)\right)=1,
$$

which is equivalent to

$$
\cos (\phi)=\cos \left(\theta_{i}^{(i)}\right)+\frac{z \lambda}{M D},
$$

where $z \in \mathbb{Z}$, such that $\cos (\phi) \in[-1,1]$ and $\phi \notin I_{i}^{(i)}$. Fig. 2 depicts the behavior of $J\left(\theta_{i}^{(i)}, \phi\right)$ when $\theta_{i}^{(i)}=0$ and for various values of $M$, where $J\left(\theta_{i}^{(i)}, \phi\right)$ is computed numerically for all values of $\phi$ using (14). It can be observed that the number of zeros of the function $J\left(\theta_{i}^{(i)}, \phi\right)$ depends on the number of antennas and it is equal to $M-1$.

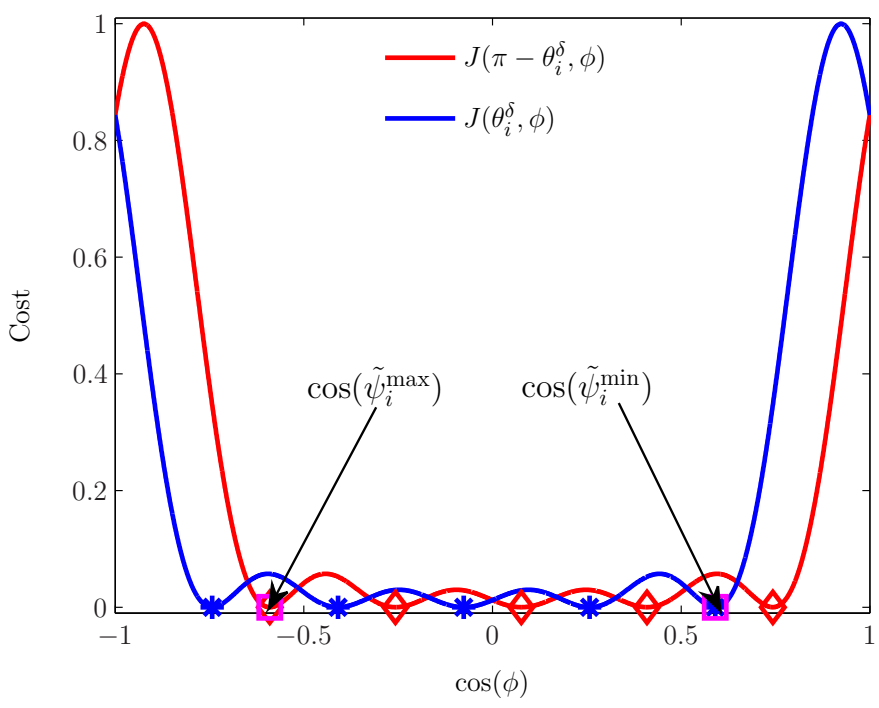

Fig. 3. The behavior of the cost functions $J\left(\theta_{i}^{\delta}, \phi\right)$ and $J\left(\pi-\theta_{i}^{\delta}, \phi\right)$ for the values of $\cos (\phi)=[-1,1], M=6$, and $\theta_{i}^{\delta}=\frac{\pi}{8}$. Red diamonds and blue asterisks represent the zeros of the functions $J\left(\theta_{i}^{\delta}, \phi\right), J\left(\pi-\theta_{i}^{\delta}, \phi\right)$, respectively. Purple squares denote $\tilde{\psi}_{i}^{\min }$ and $\tilde{\psi}_{i}{ }^{\max }$.

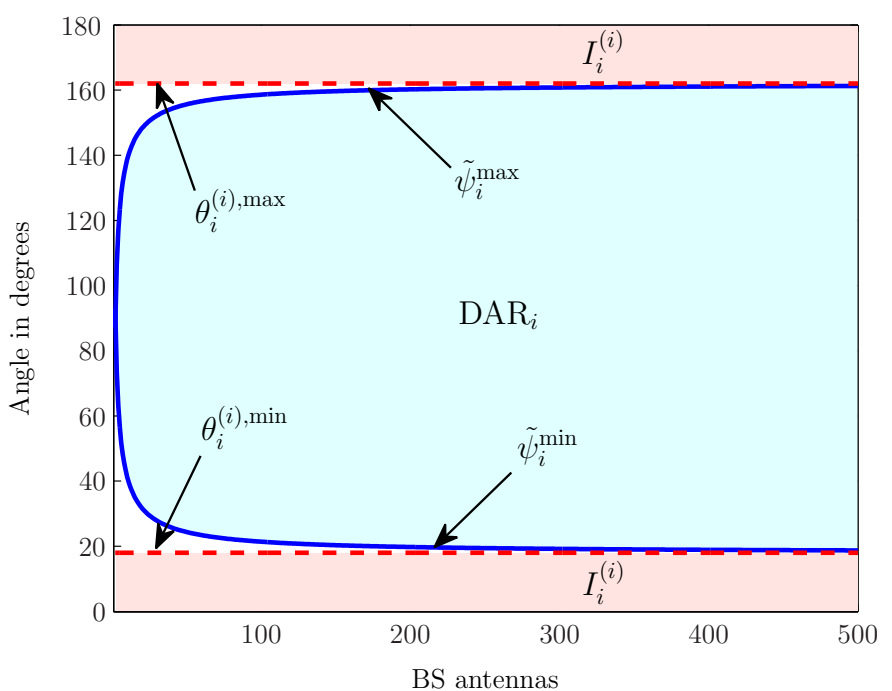

Fig. 4. The relation between $\tilde{\psi}_{i}{ }^{\min }$ to $\theta_{i}^{(i), \min }$ and $\tilde{\psi}_{i}^{\max }$ to $\theta_{i}^{(i), \max }$ with increasing BS antennas for $\theta_{i}^{\delta}=\frac{\pi}{10}$. Asymptotically, when $M \rightarrow \infty, \tilde{\psi}_{i}{ }^{\text {min }}$ converges to $\theta_{i}^{(i), \min }$ and $\tilde{\psi}_{i}{ }^{\max }$ to $\theta_{i}^{(i), \max }$. The shaded regions are: $\mathrm{DAR}_{i}$ (blue) and $I_{i}^{(i)}$ (red).

To ensure limited impact of user $j$ on user $i$, (12c) should be small for all $\phi \in I_{j}^{(i)}$ and all $\theta_{i}^{(i)} \in I_{i}^{(i)}$. Hence, we consider $J\left(\theta_{i}^{(i)}, \phi\right)$ for $\theta_{i}^{(i)}$ at the boundaries of $I_{i}^{(i)}$, i.e., $\theta_{i}^{(i)}=\theta_{i}^{\delta}$ and $\theta_{i}^{(i)}=\pi-\theta_{i}^{\delta}$, as shown in Fig. 3. We observe that both $J\left(\theta_{i}^{\delta}, \phi\right)$ and $J\left(\pi-\theta_{i}^{\delta}, \phi\right)$ are small for $\cos (\phi) \in\left[\cos \left(\tilde{\psi}_{i}^{\min }\right), \cos \left(\tilde{\psi}_{i}{ }^{\max }\right)\right]$, where the values of $\tilde{\psi}_{i}{ }^{\min }$ and $\widetilde{\psi}_{i}$ max are detailed in Appendix $\mathrm{C}$ and visualized in Fig. 3.

Therefore, when the AoA support $I_{j}^{(i)}$ of the interfering user $j$ lies within the desired angular region $\left(\mathrm{DAR}_{i}\right)\left[\tilde{\psi}_{i}^{\min }, \tilde{\psi}_{i}^{\max }\right]$ of the target user $i$ then the interference is limited. This property is exploited to devise various coordinated pilot assignment schemes in Section V. As it is observed in Fig. 2 , the range of the $\mathrm{DAR}_{i}$ increases with $\mathrm{BS}$ antennas. The relation between $\tilde{\psi}_{i}{ }^{\min }, \theta_{i}^{(i) \text {, min }}$ and $\tilde{\psi}_{i}{ }^{\max }, \theta_{i}^{(i), \max }$ with $M$ is depicted in Fig. 4. Asymptotically, when $M \rightarrow \infty$, we note that $\lim _{M \rightarrow \infty} \tilde{\psi}_{i}^{\min }=\theta_{i}^{(i), \min }=\theta_{i}^{\delta}$ and $\lim _{M \rightarrow \infty} \tilde{\psi}_{i}^{\max }=$ $\theta_{i}^{(i), \max }=\pi-\theta_{i}^{\delta}$. In [14], only for the case $M \rightarrow \infty$, the AoA condition that needs to be satisfied between interfering and target users is provided, while Fig. 4 complements this for finite $M$.

2) Approximation of cost function: Instead of storing cost functions $J\left(\theta_{i}^{(i)}, \phi\right), \forall \theta_{i}^{(i)} \in I_{i}^{(i)}$ for ev- 


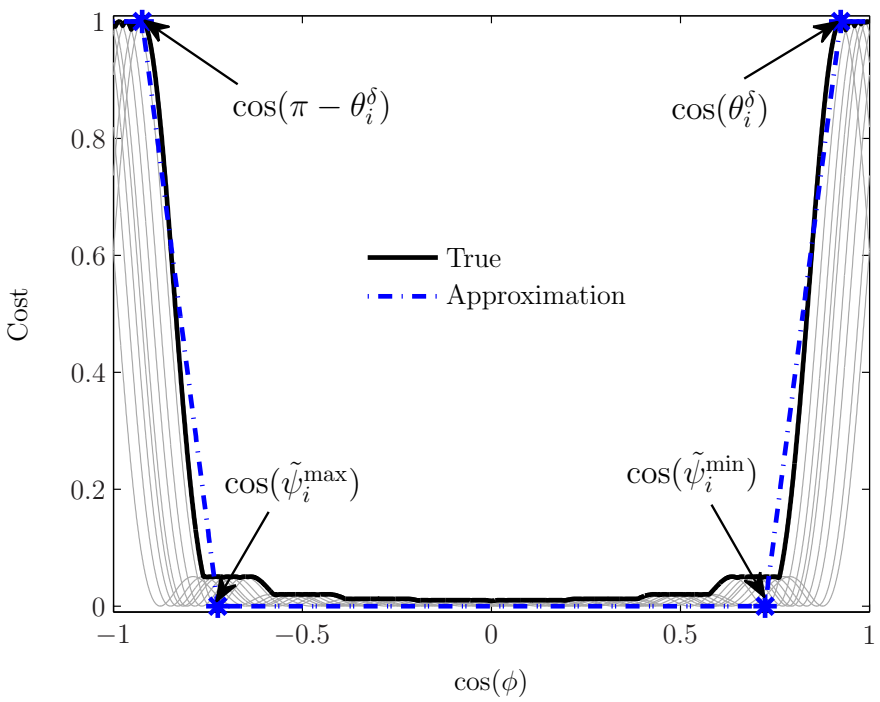

Fig. 5. The comparison between true cost function $J^{\text {True }}(\phi)$ and the approximate cost function $J^{\mathrm{Apprx}}(\phi)$ for $M=10$ and $\theta_{i}^{\delta}=\frac{\pi}{8}$. The approximate cost function $J^{\operatorname{Apprx}}(\phi)$ is a piece wise linear function connecting the following poins $(-1,1),(\cos (\pi-$ $\left.\left.\theta_{i}^{\delta}\right), 1\right),\left(\cos \left(\tilde{\psi}_{i}^{\max }\right), 0\right),\left(\cos \left(\tilde{\psi}_{i}^{\min }\right), 0\right),\left(\cos \left(\theta_{i}^{\delta}\right), 1\right),(1,1)$. The thin grey lines represent the cost functions $J\left(\theta_{i}^{(i)}, \phi\right), \forall \theta_{i}^{(i)} \in I_{i}^{(i)}$.

ery value of $\phi$, we define the combined cost function $J^{\text {True }}(\phi)=\max _{\theta_{i}^{(i)} \in I_{i}^{(i)}} J\left(\theta_{i}^{(i)}, \phi\right)$. The individual cost functions $J\left(\theta_{i}^{(i)}, \phi\right)$ and the combined cost function $J^{\text {True }}(\phi)$ is shown in Fig. 5. The cost function $J^{\text {True }}(\phi)$ can be approximated by a piecewise linear function $J^{\text {Apprx }}(\phi)$ (See blue dotted line in Fig. 5):

$$
\begin{aligned}
& J^{\operatorname{Apprx}}(\phi)= \\
& \begin{cases}1, & \cos (\phi) \leq \cos \left(\pi-\theta_{i}^{\delta}\right), \\
1-\frac{\cos (\phi)+\cos \left(\theta_{i}^{\delta}\right)}{\cos \left(\tilde{\psi}_{i}^{\max }\right)+\cos \left(\theta_{i}^{\delta}\right)}, & -\cos \left(\theta_{i}^{\delta}\right) \leq \cos (\phi) \leq \cos \left(\tilde{\psi}_{i}^{\max }\right), \\
\frac{\cos (\phi)-\cos \left(\tilde{\psi}_{i}^{\min }\right)}{\cos \left(\theta_{i}^{\delta}\right)-\cos \left(\tilde{\psi}_{i}^{\min }\right)}, & \cos \left(\tilde{\psi}_{i}^{\min }\right) \leq \cos (\phi) \leq \cos \left(\theta_{i}^{\delta}\right), \\
1, & \cos (\phi) \geq \cos \left(\theta_{i}^{\delta}\right), \\
0, & \text { elsewhere. }\end{cases}
\end{aligned}
$$

Based on the $J^{\text {Apprx }}(\phi)$, we define $J_{i j}$ as the interference cost to the BS of user $i$ experiences from another user $j$, which basically assigns zero cost when $I_{j}^{(i)}$ lies within the $\mathrm{DAR}_{i}$ of user $i$. Outside the $\mathrm{DAR}_{i}$, the cost grows linearly and saturates to unity at $\theta_{i}^{(i), \min }$ and $\theta_{i}^{(i), \max }$. For the interfering user $j$ with $I_{j}^{(i)}=\left[\theta_{j}^{(i), \min }, \theta_{j}^{(i), \max }\right], \theta_{j}^{(i), \text { min }}<\theta_{j}^{(i), \max }$, we can write $J_{i j}=J^{\operatorname{Apprx}}\left(\theta_{j}^{(i), \min }\right)+J^{\operatorname{Apprx}}\left(\theta_{j}^{(i), \max }\right)$. The interference cost $J_{i j}$ is used in devising coordinated pilot assignment schemes described in Section V.

\section{Coordinated Pilot Assignment Schemes}

In this section, we show different user assignment strategies for pilot decontamination under various configurations based on the theory developed in Section IV.

\section{A. Multi-User Multi-Cell Optimization}

For this scenario, the goal is to reuse the pilots among the users in the best possible way such that each user has been allocated a pilot. Let us collect the users from all the cells in a set $\mathcal{N}=\cup_{j \in \mathcal{C}} \mathcal{K}_{j}$, where $\mathcal{C}$ denotes the set of all cells and $\mathcal{K}_{j}$ the set of users in the $j$-th cell, $j \in \mathcal{C}$. Recall $\mathcal{P}$ be the all available orthogonal pilot sequences. Let us introduce the variable $y_{i p} \in\{0,1\}$. The variable $y_{i p}=1$, if $i$-th user is activated on $p$-th pilot and 0 otherwise. The one-shot joint optimization for user assignment for multi-user and multi-cell scenario can be written as a binary integer program (BIP):

$$
\begin{aligned}
\operatorname{minimize} & \sum_{p \in \mathcal{P}} \sum_{i \in \mathcal{N}} \sum_{j \neq i} U_{i j} y_{i p} y_{j p} \\
& \sum_{p} y_{i p} \geq 1, \forall i \in \mathcal{N} \\
& y_{i p} \in\{0,1\}, \forall i \in \mathcal{N}, p \in \mathcal{P},
\end{aligned}
$$

where

$$
U_{i j}= \begin{cases}J_{i j}, & i \neq j, \\ 0, & i=j,\end{cases}
$$

We note the following: (18a) gives preference to users who lie within the desired angular region of the target user; (18b) states each user must be active at least on one pilot; and (18c) imposes the binary integer requirements on the optimization variables. For each pilot, the optimization (18), looks for users in each cell with users in every cell and then chooses a set of users such that when assigned the same pilot to them, they will have minimum possible interference at their respective BSs.

The user assignment is performed based on the location for a target user, say $i$, accounting for the following cases:

(C1) The support of interfering signals AoA $I_{j}^{(i)}, \forall j$ lies completely inside the $\mathrm{DAR}_{i}$ of the target user;

(C2) The support of interfering signal AoA $I_{j}^{(i)}, \forall j$ lies partially inside the $\mathrm{DAR}_{i}$ of the target user; and

(C3) The support of interfering signal AoA $I_{j}^{(i)}, \forall j$ lies completely outside with the $\mathrm{DAR}_{i}$ of the target user.

The above optimization problem is always feasible. The problem (18) gives preference to users that satisfy the case (C1), in which case the objective is zero. It might be possible that the user locations are such that (C1) cannot be satisfied. This is tackled in (18), as it implicitly considers the cases (C2) and (C3) in the formulation. For example, when $I_{j}^{(i)}$ does not lie within $\mathrm{DAR}_{i}$, then the objective function becomes positive. Therefore, to minimize the objective, the interfering users are selected in such a way that the maximal overlap with the desired support of the target user is obtained.

The optimization (18) can be written as an integer quadratic constraint optimization problem (IQCP) as

$$
\begin{aligned}
\operatorname{minimize} & \mathbf{y}^{\mathrm{T}} \mathbf{Q} \mathbf{y} \\
& \sum y_{i p} \geq 1, \forall i \in \mathcal{N} \\
& y_{i p} \in\{0,1\}, \forall i \in \mathcal{N}, p \in \mathcal{P},
\end{aligned}
$$

where $\mathbf{y}=\left[y_{11}, \ldots, y_{|\mathcal{N}| 1}, \ldots, y_{1|\mathcal{P}|}, \ldots, y_{|\mathcal{N}||\mathcal{P}|}\right], \mathbf{Q}$ is a $|\mathcal{N}||\mathcal{P}| \times|\mathcal{N}||\mathcal{P}|$ block diagonal matrix given by

$$
\mathbf{Q}=\left[\begin{array}{cccc}
\mathbf{U} & \mathbf{0}_{|\mathcal{N}|} & \cdots & \mathbf{0}_{|\mathcal{N}|} \\
\mathbf{0}_{|\mathcal{N}|} & \mathbf{U} & \cdots & \mathbf{0}_{|\mathcal{N}|} \\
\vdots & \vdots & \ddots & \vdots \\
\mathbf{0}_{|\mathcal{N}|} & \mathbf{0}_{|\mathcal{N}|} & \cdots & \mathbf{U}
\end{array}\right]
$$


where $\mathbf{0}_{|\mathcal{N}|}$ denotes a square matrix of dimension $|\mathcal{N}| \times|\mathcal{N}|$ with zero entries, and $\mathbf{U}$ is the $|\mathcal{N}| \times|\mathcal{N}|$ dimension utility matrix with entries

$$
\mathbf{U}=\left[\begin{array}{cccc}
U_{11} & U_{12} & \cdots & U_{1|\mathcal{N}|} \\
U_{21} & U_{22} & \cdots & U_{2|\mathcal{N}|} \\
\vdots & \vdots & \ddots & \vdots \\
U_{|\mathcal{N}| 1} & U_{|\mathcal{N}| 2} & \cdots & U_{|\mathcal{N}||\mathcal{N}|}
\end{array}\right]
$$

where $U_{i j}$ is given in (19).

Remark 3. The optimization problem (18) is formulated as an IQCP and it can be easily shown to be $\mathcal{N} \mathcal{P}$-hard. It should be also emphasized that the above optimization problem is dependent on the number of antennas at each BS via $U_{i j}$ (see (19)).

The formulation of the optimization problem (20) is very general and encompasses a multitude of possible scenarios even when the users for assignment belong to the same cell as that of the target user. In what follows, we show some variants of interest of (20) in the subsequent sections.

\section{B. Multi-User Multi-Cell Optimization with QoS Guarantees}

For the sake of establishing some QoS guarantees, we may want to exclude the possibility of assigning users from the same cell as that of the target user to the same pilot. This is achieved by changing (20b) to a constraint such that only one user from each cell is assigned per pilot; this is enforced in the optimization problem via (23b). Note that if no user exists in a cell for a certain pilot, then for constraint (23b) to be valid, without loss of generality, the constraint for that cell at a certain pilot is removed. Therefore, the IQCP formulation for multi-user and multi-cell optimization with QoS guarantees is written as

$$
\begin{aligned}
\operatorname{minimize} & \mathbf{y}^{\mathrm{T}} \mathbf{Q} \mathbf{y} \\
& \sum_{i \in \mathcal{K}_{j}} y_{i p}=1, \forall j \in \mathcal{C}, p \in \mathcal{P} \\
& y_{i p} \in\{0,1\}, \forall i \in \mathcal{N}, p \in \mathcal{P} .
\end{aligned}
$$

\section{Multi-User Single-Cell Optimization}

Our objective is to find for each user in a target cell one user from the neighboring cells and assign them the same pilot sequence. For this scenario, the mutual interference of the users at the respective BSs is ignored and only the interference of the users observed at the target cell are required to be satisfied. This scenario finds applications to cases where priority is required to be given to a certain cell (for example, in case there is a special event that requires wireless communications to be robust) and in dense urban areas where it is computationally very expensive to include all the cells in the network and inevitably to run such a large-scale optimization in real time.

The set of users for this scenario are the users from the target cell and its neighboring cells. Let the set of users given by $\mathcal{M}=\mathcal{K}_{q} \cup\left(\cup_{i \in \mathcal{C}_{q}^{\text {sur }}} \mathcal{K}_{i}\right)$, where $\mathcal{C}_{q}^{\text {sur }}$ is the set of cells surrounding cell $q$. The modified optimization is then written
TABLE I

SimUlation PARAMETERS

\begin{tabular}{|c|c|c|c|}
\hline Parameter & Value & Parameter & Value \\
\hline \hline$\eta$ & 2.5 & $r_{\mathrm{S}}$ & $50 \mathrm{~m}$ \\
\hline$\lambda$ & $0.1 \mathrm{~m}$ & $\gamma_{\mathrm{SNR}}$ & $20 \mathrm{~dB}$ \\
\hline$\sigma^{2}$ & 0.001 & $B$ & 50 \\
\hline$R$ & $1000 \mathrm{~m}$ & $\tau$ & 10 \\
\hline$D$ & $\lambda / 2$ & & \\
\hline \multicolumn{3}{|r}{} \\
\hline
\end{tabular}

as

$$
\begin{aligned}
\operatorname{minimize} & \mathbf{y}^{\mathrm{T}} \overline{\mathbf{Q}} \mathbf{y} \\
& \sum_{i \in \mathcal{K}_{j}} y_{i p}=1, \forall j \in\left(q \cup \mathcal{C}_{q}^{\text {sur }}\right), p \in \mathcal{P} \\
& y_{i p} \in\{0,1\}, \forall i \in \mathcal{M}, p \in \mathcal{P},
\end{aligned}
$$

where $\overline{\mathbf{Q}}$ is a $|\mathcal{M}||\mathcal{P}| \times|\mathcal{M} \| \mathcal{P}|$ block diagonal matrix given by

$$
\overline{\mathbf{Q}}=\left[\begin{array}{cccc}
\overline{\mathbf{U}} & \mathbf{0}_{|\mathcal{M}|} & \cdots & \mathbf{0}_{|\mathcal{M}|} \\
\mathbf{0}_{|\mathcal{M}|} & \overline{\mathbf{U}} & \cdots & \mathbf{0}_{|\mathcal{M}|} \\
\vdots & \vdots & \ddots & \vdots \\
\mathbf{0}_{|\mathcal{M}|} & \mathbf{0}_{|\mathcal{M}|} & \cdots & \overline{\overline{\mathbf{U}}}
\end{array}\right]
$$

where $\overline{\mathbf{U}}$ is the $|\mathcal{M}| \times|\mathcal{M}|$ dimension utility matrix with entries

$$
\bar{U}_{i j}= \begin{cases}U_{i j} & , \text { if } i \in \mathcal{K}_{q} \\ 0 & , \text { otherwise }\end{cases}
$$

\section{Numerical Results}

In this section, we present numerical results to evaluate the proposed schemes described in Section V. We consider hexagonal shaped cells and simulations are performed with a multi-cell system scenario. The simulation parameters used to obtain the numerical results are given in Table I. We keep these parameters fixed for the simulations unless otherwise specified.The performance metric considered for the numerical results is the normalized channel estimation error $\mathcal{E}$, which is defined in $\mathrm{dB}$ scale as

$$
\mathcal{E}_{k}[\mathrm{~dB}]=10 \log _{10}\left(\frac{\left\|\hat{\mathbf{h}}_{i k k}-\mathbf{h}_{i k k}\right\|_{\mathrm{F}}^{2}}{\left\|\mathbf{h}_{i k k}\right\|_{\mathrm{F}}^{2}}\right),
$$

where $\mathbf{h}_{i k k}$ and $\hat{\mathbf{h}}_{i k k}$ are the desired and estimated channels of user $i$ at the $k$-th $\mathrm{BS}$ in $k$-th cell.

\section{A. Impact of Different AoA Supports of an Interfering User}

In Fig. 6, we show the impact of different ranges of AoA support $I_{j}^{(i)}$ of a single interfering user w.r.t. to $\mathrm{DAR}_{i}$ of the target user. Note that all the angles are after the angular transformation performed w.r.t. to the target user. We can calculate $\mathrm{DAR}_{i}$, and for $M=10$, it is obtained as $\left[37.6^{\circ}, 142.4^{\circ}\right]$. We varied $I_{j}^{(i)}$ such that some are within $\mathrm{DAR}_{i}$ and some are outside this range. When $I_{j}^{(i)}$ lies within $\mathrm{DAR}_{i}$ then the interference is low, and the channel estimation converges fast to the interference-free scenario; with $M=10 \mathrm{BS}$ antennas the channel estimation performance is similar to that of the 


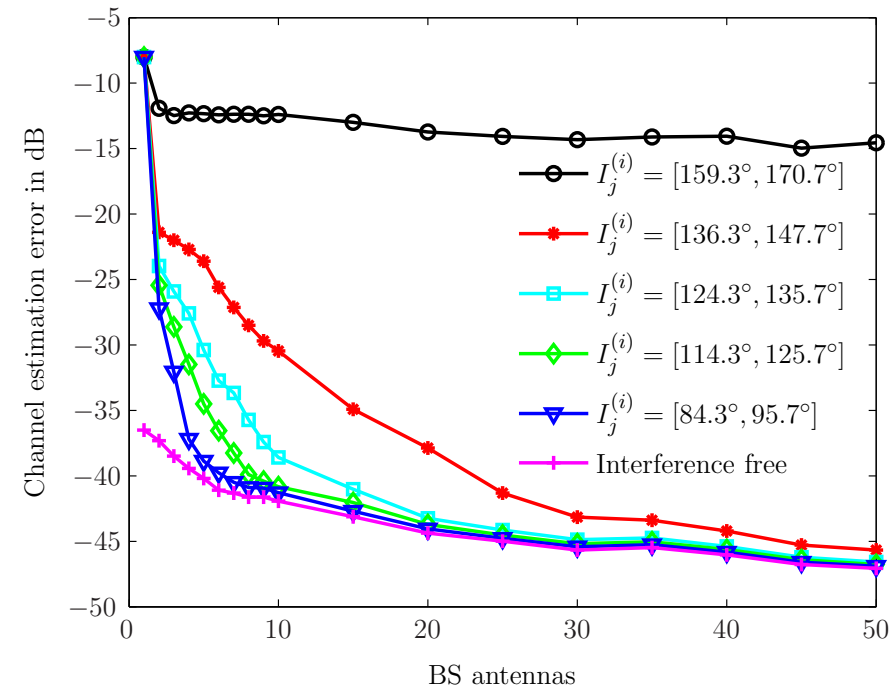

Fig. 6. Comparison of channel estimation error $\mathcal{E}$ versus BS antennas for different AoA $I_{j}^{(i)}$ of single interfering user. The target user is placed in $d=500 \mathrm{~m}$ and the interfering user is placed in $d=1000 \mathrm{~m}$ from the target BS. The results are averaged over 1000 Monte-Carlo channel realizations.

interference-free scenario. On the other hand, when $I_{j}^{(i)}$ is outside the $\mathrm{DAR}_{i}$, it can be observed that more BS antennas are needed to converge to the interference-free scenario. For example, when $I_{j}^{(i)}=\left[136.3^{\circ} 147.7^{\circ}\right]$, then more than $50 \mathrm{BS}$ antennas are required.

\section{B. Impact of the Distance of an Interfering User}

The impact of the various interferer distances $d=$ $500,1000,1500,2000 \mathrm{~m}$ on the channel estimation performance is shown in Fig. 7. Again, for $M=10$, we have $\operatorname{DAR}_{i}=\left[37.6^{\circ}, 142.4^{\circ}\right]$. The impact of the interferer distance is very significant, when interferer $\mathrm{AoA}\left(\operatorname{see} \theta_{j}^{(i), \mu}=150^{\circ}\right.$ ) lies outside the $\mathrm{DAR}_{i}$ of the target user. The channel estimation performance is deteriorated when the interferer distance is decreased. On the other hand, when interferer AoA (see $\theta_{j}^{(i), \mu}=60^{\circ}$ and $\theta_{j}^{(i), \mu}=90^{\circ}$ ) lies inside the $\mathrm{DAR}_{i}$ of the target user, the impact of distance is not significant. Unless, when the interferer is also from the target cell, i.e., $d=500$ m.

\section{Greedy vs Joint Optimization of Users}

In Fig. 8, we show the advantage of the proposed joint optimization scheme (see optimization (24)) over the greedy sequential user assignment [14], [15]. We considered a twocell scenario which consists of two users in each cell (see Fig. 8 (a)). Also, there are two pilots to be assigned for the two users in cell-1. Both approaches try to find for each pilot one user from cell-1 and assign with another user from cell-2. For this scenario, both the users from cell-2 fall within the desired angular region of user-1 in cell-1. However, for user-2 of cell1 , only user- 1 of cell- 2 is permissible. In the greedy sequential scheme, user-1 of cell-1 is assigned with user- 1 of cell-2, as it is the best two users because of the maximum angular separation. However, for the joint optimization, it considers

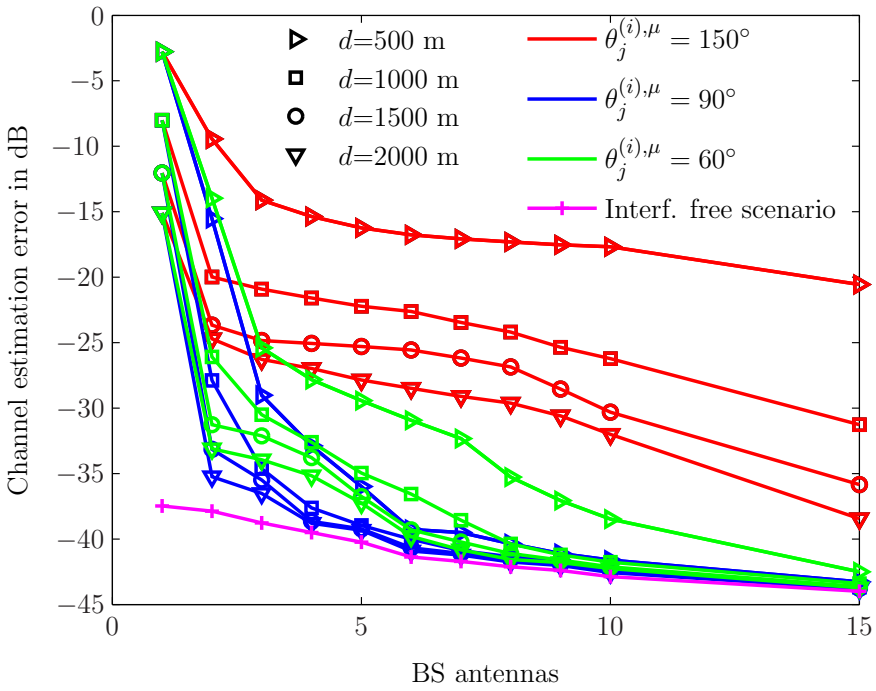

Fig. 7. Comparison of channel estimation error $\mathcal{E}$ versus BS antennas for different mean $\operatorname{AoA}\left(\tilde{\theta}_{j}^{(i), \mu}\right)$ and for different distances of a single interfering user. For this setup, the target user is placed at $d=500 \mathrm{~m}$ from the target BS. The spread of AoA $\theta^{\delta}$ of the users is calculated based on its distance from the target BS with $r_{\mathrm{S}}=100 \mathrm{~m}$. The results are averaged over 1000 Monte-Carlo channel realizations.

compatibility of both users of cell- 1 . Therefore, it assigns user1 of cell- 1 with user- 2 of cell- 2 and user- 2 of cell- 1 with user- 1 of cell-2. The channel estimation error $\mathcal{E}$ performance for both schemes is shown in Fig. 8 (b). As expected, the performance of pilot-2 with greedy assignment suffers as user- 2 of cell- 1 is not compatible with user- 2 of cell-2.

\section{Multi-cell Mutual Interference Scenario}

The channel estimation performance of the target user in each cell for a two-cell scenario is depicted in Fig. 9. The users are assigned to a pilot based on the multi-user multicell optimization (20). Users in different cells are assigned the same pilot if the AoA support of a user in one cell lies within the desired angular region of another user in another cell and vice-versa. Consequently, the channel estimation performance of the target users at their respective BS is improved.

\section{CONClusions And Future Directions}

In this paper, we characterized the effect of interference for MIMO BSs with a large, but finite, number of antennas, harnessing user location information. Building on this characterization, we formulated several pilot assignment problems as integer quadratic constraint optimization problem. These problems are solved centrally, provided that all the information about all users is shared among all BSs. We show that the pilot contamination for the proposed pilot assignment strategies is eliminated even when the number of antennas is finite.

The centralized approach proposed in the paper is computationally expensive and requires high communication overhead. Part of our ongoing research focuses on developing distributed implementations based on local information only. 


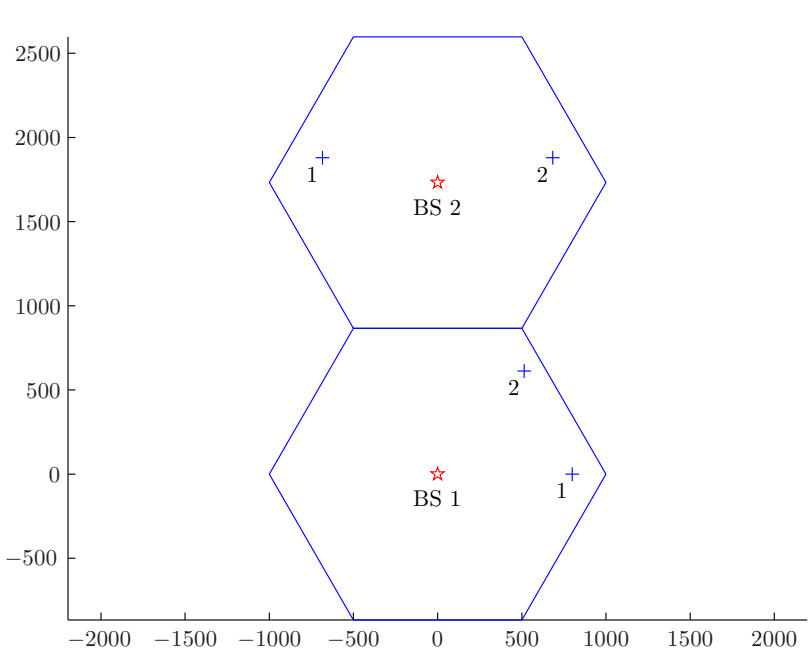

(a)

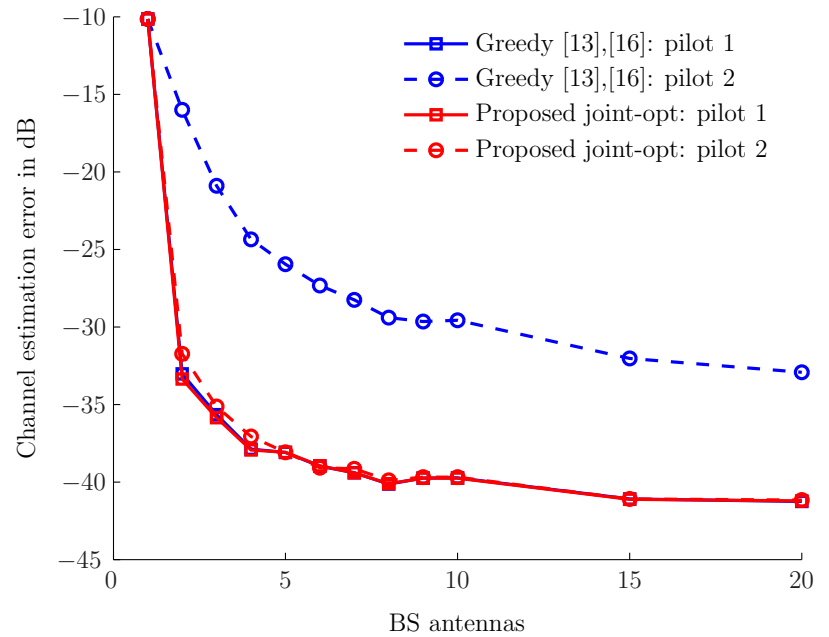

(b)

Fig. 8. Performance comparison of a greedy vs joint optimization for a two cell scenario. Inset (a) two cell scenario with two users each, users are marked with a plus sign and BS with pentagons, and (b) channel estimation error $\mathcal{E}$ with BS antennas for the users with greedy and joint optimization schemes.

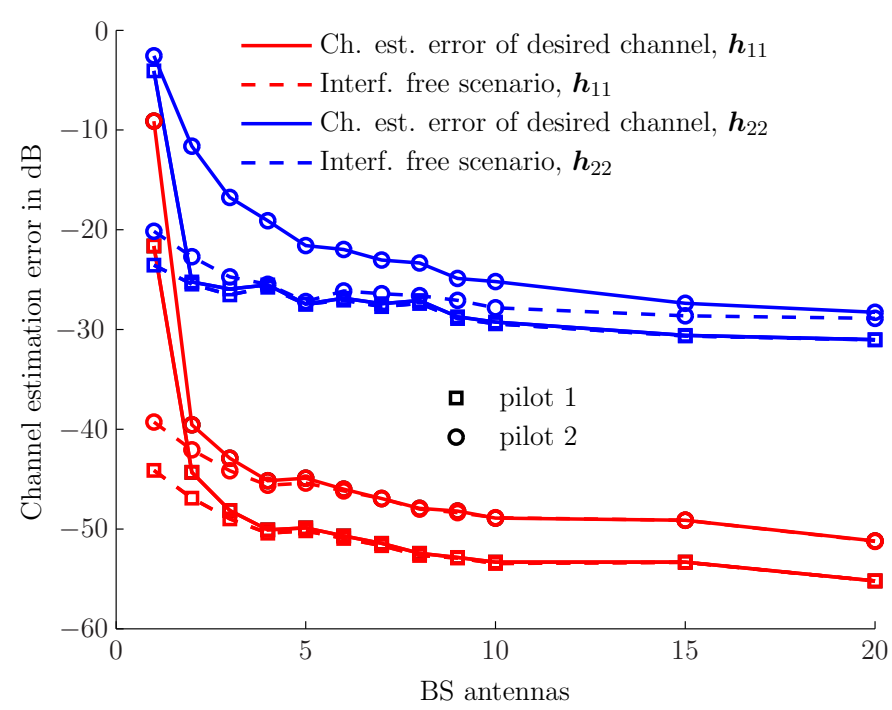

Fig. 9. Channel estimation performance of the target users in each cell for a two-cell scenario.

\section{APPENDIX A}

\section{MOTIVATION FOR CLAIMS}

The channel estimate $\hat{\mathbf{h}}_{i}$ of the desired user can be written using (4) and (5) as

$$
\begin{aligned}
\hat{\mathbf{h}}_{i} & =\mathbf{R}_{i}\left(\sigma^{2} \mathbf{I}_{M}+\tau \sum_{j=1}^{L} \mathbf{R}_{j}\right)^{-1} \overline{\mathbf{S}^{\mathrm{H}}}\left(\overline{\mathbf{S}} \sum_{j=1}^{L} \mathbf{h}_{j}+\mathbf{n}\right), \\
& =\mathbf{R}_{i}\left(\sigma^{2} \mathbf{I}_{M}+\tau \sum_{j=1}^{L} \mathbf{R}_{j}\right)^{-1}\left(\tau \sum_{j=1}^{L} \mathbf{h}_{j}+\overline{\mathbf{S}}^{\mathrm{H}} \mathbf{n}\right) .
\end{aligned}
$$

We expand

$$
\mathbf{I}_{M}=\mathbf{V} \mathbf{V}^{\mathrm{H}}+\sum_{j=1}^{L} \mathbf{U}_{j} \mathbf{U}_{j}^{\mathrm{H}},
$$

where $\mathbf{U}_{j}$ is a unitary matrix of size $M \times m_{j}$ and $\mathbf{V}$ is a unitary matrix such that (29) holds. By decomposing covariance matrices $\mathbf{R}_{j}=\mathbf{U}_{j} \Sigma_{j} \mathbf{U}_{j}^{\mathrm{H}}, \forall j$, where $\Sigma_{j}$ is an eigenvalue matrix of size $m_{j} \times m_{j}$, (28) becomes

$$
\begin{aligned}
\hat{\mathbf{h}}_{i} & =\sum_{j \neq i}^{L} \mathbf{R}_{i} \mathbf{U}_{j}\left(\sigma^{2} \mathbf{I}_{m_{j}}+\tau \Sigma_{j}\right)^{-1} \mathbf{U}_{j}^{\mathrm{H}} \tau \sum_{j^{\prime}=1}^{L} \mathbf{h}_{j^{\prime}} \\
& +\mathbf{U}_{i} \Sigma_{i}\left(\sigma^{2} \mathbf{I}_{m_{i}}+\tau \Sigma_{i}\right)^{-1}\left(\mathbf{U}_{i}^{\mathrm{H}} \sum_{j \neq i}^{L} \mathbf{h}_{j}+\mathbf{U}_{i}^{\mathrm{H}} \mathbf{h}_{i}\right) \\
& +\mathbf{U}_{i} \Sigma_{i}\left(\sigma^{2} \mathbf{I}_{m_{i}}+\tau \Sigma_{i}\right)^{-1} \mathbf{U}_{i}^{\mathrm{H}} \overline{\mathbf{S}}^{\mathrm{H}} \mathbf{n} .
\end{aligned}
$$

Hence, to ensure that $\hat{\mathbf{h}}_{i}$ is close to the interference-free estimate $\hat{\mathbf{h}}_{i}^{\text {no-int }}$, it is sufficient to have

$$
\left\|\mathbf{U}_{i}^{\mathrm{H}} \mathbf{h}_{j}\right\| \ll\left\|\mathbf{U}_{i}^{\mathrm{H}} \mathbf{h}_{i}\right\|, \forall j \neq i,
$$

and

$$
\begin{aligned}
& \left\|\mathbf{R}_{i} \mathbf{U}_{j}\left(\sigma^{2} \mathbf{I}_{m_{j}}+\tau \Sigma_{j}\right)^{-1} \mathbf{U}_{j}^{\mathrm{H}} \mathbf{h}_{j^{\prime}}\right\| \\
& \ll\left\|\mathbf{U}_{i} \Sigma_{i}\left(\sigma^{2} \mathbf{I}_{m_{i}}+\tau \Sigma_{i}\right)^{-1} \mathbf{U}_{i}^{\mathrm{H}} \mathbf{h}_{i}\right\|, \forall j \neq i, \forall j^{\prime} .
\end{aligned}
$$

Since $\mathbf{h}_{j}$ lies in the span of the steering vectors of user $j$, rendering $\mathbf{a}^{\mathrm{H}}\left(\theta_{j}\right) \mathbf{R}_{i} \mathbf{a}\left(\theta_{j}\right)$ small for all $\theta_{j} \in I_{j}^{(i)}$ will result in meeting condition (30). Condition (31) can be met when the columns of $\mathbf{U}_{j}$ are close to the null-space of $\mathbf{R}_{i}$. Since the span of the steering vectors of any user $j$ is a super-set of the range of $\mathbf{R}_{j}$ and thus of $\mathbf{U}_{j}$, rendering $\mathbf{a}^{\mathrm{H}}\left(\theta_{j}\right) \mathbf{R}_{i} \mathbf{a}\left(\theta_{j}\right)$ small for all $\theta_{j} \in I_{j}^{(i)}$ will thus also result in meeting condition (31). In summary, interfering users for which $\mathbf{a}^{H}\left(\theta_{j}\right) \mathbf{R}_{i} \mathbf{a}\left(\theta_{j}\right)$ is small are preferred to limit the impact of interference during channel estimation.

\section{APPENDIX B PROOF OF PROPOSITION 2}

$$
\begin{aligned}
& \min _{\phi \in \Phi} \mathbf{u}^{\mathrm{H}} \mathbf{R}_{i} \mathbf{u} \\
& =\min _{\phi \in \Phi} \frac{1}{M} \int J^{2}\left(\theta_{i}^{(i)}, \phi\right) p\left(\theta_{i}^{(i)}\right) d \theta_{i}^{(i)}, \\
& \stackrel{(a)}{=} \frac{1}{M} \int \min _{\phi \in \Phi} J^{2}\left(\theta_{i}^{(i)}, \phi\right) p\left(\theta_{i}^{(i)}\right) d \theta_{i}^{(i)},
\end{aligned}
$$


where for $(a)$ we make use of [18, Theorem 14.60], which states that the interchange of minimization and integration is possible under certain conditions; in our case, the conditions are met since the integrand is continuous in $\theta_{i}^{(i)}$ and the minimization is over the space of all measurable functions and hence it can be reduced to point-wise minimization.

\section{APPENDIX C
SELECTION OF $\tilde{\psi}_{i}^{\text {min }}$ AND $\tilde{\psi}_{i}^{\max }$}

We denote $\left\{\phi_{r, \theta_{i}^{\delta}}^{*}\right\}_{r}$ and $\left\{\phi_{r, \pi-\theta_{i}^{\delta}}^{*}\right\}_{r}$ as the sets of zeros of functions $J\left(\theta_{i}^{\delta}, \phi\right)$ and $J\left(\pi-\theta_{i}^{\delta}, \phi\right)$, respectively. The values of $\phi_{\theta_{i}^{\delta}}^{*}$ and $\phi_{\pi-\theta_{i}^{\delta}}^{*}$ are obtained by solving the following expressions using (16)

$$
\begin{aligned}
\cos \left(\phi_{\theta_{i}^{\delta}}^{*}\right) & =\cos \left(\theta_{i}^{\delta}\right)+\frac{z \lambda}{M D}, \\
\cos \left(\phi_{\pi-\theta_{i}^{\delta}}^{*}\right) & =-\cos \left(\theta_{i}^{\delta}\right)+\frac{z \lambda}{M D} .
\end{aligned}
$$

$$
\begin{aligned}
& \text { We further define } \tilde{\psi}_{i}^{\min } \\
& \max \left(\min _{r}\left(\left\{\phi_{r, \theta_{i}^{\delta}}^{*}\right\}_{r}\right), \min _{r}\left(\left\{\phi_{r, \pi-\theta_{i}^{\delta}}^{*}\right\}_{r}\right)\right) \text { and } \tilde{\psi}_{i} \text { max }= \\
& \min \left(\max _{r}\left(\left\{\phi_{r, \theta_{i}^{\delta}}^{*}\right\}_{r}\right), \max \left(\left\{\phi_{r, \pi-\theta_{i}^{\delta}}^{*}\right\}_{r}\right)\right) .
\end{aligned}
$$

\section{ACKNOWLEDGMENT}

We would like to thank Haifan Yin and Prof. David Gesbert for fruitful discussions. The authors would like to thank GUROBI for the free academic license to use their numerical optimization software.

\section{REFERENCES}

[1] F. Boccardi, R. W. Heath, A. Lozano, T. L. Marzetta, and P. Popovski, "Five disruptive technology directions for 5G," IEEE Communications Magazine, vol. 52, no. 2, pp. 74-80, Feb. 2014.

[2] Y.-G. Lim, C.-B. Chae, and G. Caire, "Performance analysis of massive mimo for cell-boundary users," IEEE Transactions on Wireless Communications, vol. 14, no. 12, pp. 6827-6842, Dec 2015.

[3] E. G. Larsson, O. Edfors, F. Tufvesson, and T. L. Marzetta, "Massive MIMO for next generation wireless systems," IEEE Communications Magazine, vol. 52, no. 2, pp. 186-195, Feb. 2014.
[4] F. Rusek, D. Persson, B. K. Lau, E. G. Larsson, T. L. Marzetta, O. Edfors, and F. Tufvesson, "Scaling up MIMO: Opportunities and challenges with very large arrays," IEEE Signal Processing Magazine, vol. 30, no. 1, pp. 40-60, Jan. 2013.

[5] E. Bjornson, E. G. Larsson, and M. Debbah, "Optimizing multi-cell massive MIMO for spectral efficiency: How many users should be scheduled?" in Proc. IEEE Global Conference on Signal and Information Processing, Dec. 2014, pp. 612-616.

[6] T. L. Marzetta, "Noncooperative cellular wireless with unlimited numbers of base station antennas," IEEE Transactions on Wireless Communications, vol. 9, no. 11, pp. 3590-3600, Nov. 2010.

[7] J. Hoydis, S. Ten Brink, and M. Debbah, "Massive MIMO in the UL/DL of cellular networks: How many antennas do we need?" IEEE Journal on Selected Areas in Communications, vol. 31, no. 2, pp. 160-171, 2013.

[8] H. Q. Ngo and E. G. Larsson, "EVD-based channel estimation in multicell multiuser MIMO systems with very large antenna arrays," in Proc. IEEE International Conference on Acoustics, Speech and Signal Processing, Mar. 2012, pp. 3249-3252.

[9] V. Saxena, G. Fodor, and E. Karipidis, "Mitigating pilot contamination by pilot reuse and power control schemes for massive MIMO systems," in Proc. IEEE Vehicular Technology Conference Spring, May 2015.

[10] K. Appaiah, A. Ashikhmin, and T. L. Marzetta, "Pilot contamination reduction in multi-user TDD systems," in Proc. IEEE International Conference on Communications, May 2010.

[11] W. A. W. M. Mahyiddin, P. A. Martin, and P. J. Smith, "Pilot contamination reduction using time-shifted pilots in finite massive MIMO systems," in Proc. IEEE Vehicular Technology Conference (Fall), Sep. 2014.

[12] T. X. Vu, T. A. Vu, and T. Q. S. Quek, "Successive pilot contamination elimination in multiantenna multicell networks," IEEE Wireless Communications Letters, vol. 3, no. 6, pp. 617-620, Dec. 2014.

[13] J. Zhang, B. Zhang, S. Chen, X. Mu, M. El-Hajjar, and L. Hanzo, "Pilot contamination elimination for large-scale multiple-antenna aided OFDM systems," IEEE Journal of Selected Topics in Signal Processing, vol. 8, no. 5, pp. 759-772, Oct. 2014

[14] H. Yin, D. Gesbert, M. Filippou, and Y. Liu, "A coordinated approach to channel estimation in large-scale multiple-antenna systems," IEEE Journal on Selected Areas in Communications, vol. 31, no. 2, pp. 264 273, Feb. 2013.

[15] L. S. Muppirisetty, H. Wymeersch, J. Karout, and G. Fodor, "Locationaided pilot contamination elimination for massive MIMO systems," in Proc. IEEE Global Communications Conference, Dec. 2015.

[16] J.-A. Tsai, R. Buehrer, and B. Woerner, "The impact of AOA energy distribution on the spatial fading correlation of linear antenna array," in 55th IEEE Vehicular Technology Conference (Spring), vol. 2, 2002, pp. 933-937.

[17] A. Adhikary, J. Nam, J.-Y. Ahn, and G. Caire, "Joint spatial division and multiplexing: The large-scale array regime," IEEE Transactions on Information Theory, vol. 59, no. 10, pp. 6441-6463, Oct. 2013.

[18] R. T. Rockafellar and R. J.-B. Wets, Variational analysis. Springer Science \& Business Media, 2009, vol. 317. 